\title{
Forty Years on from the Question of Referential Signals in Nonhuman Communication
}

\author{
Department of Psychology, Oakland University \\ *Corresponding author (Email: vonk@oakland.edu)
}

Jennifer Vonk

Citation - Vonk, J. (2020). Forty years on from the question of referential signals in nonhuman communication. Animal Behavior and Cognition, 7(2), 82-86. https://doi.org/10.26451/abc.07.02.01.2020

\begin{abstract}
In 1980, two groundbreaking articles were published by the team of Seyfarth, Cheney and Marler. In these papers, the question of whether vervet monkeys produced and encoded semantic information in their alarm calls set the stage for experimental explorations of animal communication and raised questions about apparent continuity with human language. In the four decades that followed, researchers hotly debated the questions of referential communication capacities in a variety of primate and non-primate animals. This special issue of Animal Behavior and Cognition presents some of that research and ongoing debate. Authors argue whether animal alarm calls amount to more than acoustic expressions of emotion states, but they also extend the legacy of those early debates by presenting many new directions for understanding the evolution of language. Last year, we lost Dorothy Cheney - a pioneer in the area of primate cognition, and this special issue is a tribute and testament to the enduring legacy of her ingenious and collaborative approach to the study of animal communication.
\end{abstract}

Keywords - referential, monkey, nonhuman communication, signals, debate

Forty years ago, Seyfarth, Cheney and Marler published a brief Science report (1980a) and a more elaborated empirical paper in Animal Behaviour (1980b) that raised the intriguing possibility that monkeys conveyed referential information in their alarm calls. I was first exposed to those papers almost twenty years later as a graduate student transitioning from studying human memory to nonhuman primate cognition. Despite having worked for several years studying Mongolian gerbils in a behavioral endocrinology lab and having a longstanding love for nonhuman animals, I had received no training in the study of nonhuman primates. My only relevant coursework as a doctoral student was a thought-provoking course in primate cognition taught by Sara Shettleworth at the University of Toronto. Around this time, I came across the studies by Seyfarth and Cheney and their colleagues and I was immediately struck by their ingenuity. Having met Robert Seyfarth when he gave a talk at the University of Toronto, I recall his kindness and generosity. However, it was the simple elegance and ecological validity of his work with Cheney that stuck with me. They were unique among their peers for conducting carefully controlled experiments in their species' natural habitat.

Sometimes neglected in the vast literature seeking evidence of theory of mind in nonhuman primates, Cheney and Seyfarth's 1990 study revealing that mother monkeys did not selectively warn only uninformed offspring of possible danger was unmistakably clever. In his commentary in this issue, Boesch (2020) highlights the value of beginning research with an appreciation for the sorts of problems animals are already solving in their natural environments and conducting experiments to understand the cognitive mechanisms underlying such behavior. This approach contrasts with that of many lab researchers who often create artificial experiments that separate the animal from the selective pressures that gave rise to their cognition but whose tests often require thousands of trials of learned associations 
and present animals with scenarios they would never have to contend with naturally (see also Eaton et al., 2017). In addition, Boesch raises concern with the external validity of studies with captive animals as he questions whether their unnatural rearing conditions render them unrepresentative of their wild counterparts. These are important questions when applied to any aspect of animal cognition, but may be particularly relevant when studying communication, which certainly depends upon the kind of group structure, learning opportunities and contextual cues that may not be replicated in captive settings.

Vocal signals are of course shaped by their relevance to the receiver. Schwartz et al. (2020) point to the importance of the distinction between sender and receiver. Fisher (2020) goes further to suggest a potentially useful dichotomy between flexibility of call production and flexibility in responses to sounds. In responding to sounds, monkeys may display a flexibility through learning that is not apparent in call production. Fisher takes this to imply that auditory learning abilities evolved prior to flexibility in vocal production. She suggests that there are fewer parallels between human language and nonhuman primate communication than we might have imagined forty years ago. McRae (2020) ponders how we might examine receiver and predator responses to determine whether alarm calls contain specific or general information about threats. Sievers and Gruber (2020) also attend to the role of learning and the extent to which it parallels human language learning. They acknowledge that Seyfarth and colleagues originally pointed out the importance of arbitrainess of the monkeys' alarm signals but that, surprisingly, the question of whether other nonhuman signals contain such arbitrary signals has not been adequately taken up by other researchers. Other directions have been more exhaustively explored.

Although the target papers (Seyfarth et al., 1980a, 1980b) are often credited with heralding a new appreciation for complexity in nonhuman communication, Snowdon (2020) reminds the reader that there were several papers published in the preceding decade that identified multiple call types in other primate and nonprimate species. In addition, he reviews a number of papers identifying other parallels between human and nonhuman communication that were of interest for the study of language evolution. Thus, it is clear, as with any profound insight, groundbreaking work does not emerge from a vaccum but rather is situated within a zeitgeist where researchers are receptive to novel ideas. However, Pepperberg (2020) rightfully reminds the reader that such groundbreaking ideas were often (and still are) met with resistence, even derision. Snowdon also reflects on the radical shift taking place at the time from the behaviorist perspective to the cognitive revolution. Pepperberg notes that, although the Seyfarth et al. papers were not alone in their insights, they were somehow better poised to inspire the leagues of young scientists prepared to take up their mantle over the past four decades than the body of work on bird communication that likely laid its foundations. The work of many of those young scientists is presented in this special issue.

One of the most notable questions to emerge from the target research is the question of how widespread referential calls might be within and outside of the primate order (e.g., McRae, 2020). Snowdon (2020) indicates that this type of referential alarm calling is actually quite unique even within primates, thus raising more questions than answers about how to reconstruct the evolution of human language. In addition, primates do not appear to make use of referential signals outside of the context of alarm calls. Snowdon singles out food calls as a promising direction but one that has apparently borne little fruit.

Snowdon (2020) and Fichtel (2020) raise the valuable insight from Wheeler and Fisher's (2012) work, that flexibility in responses to signals based on contextual cues is more interesting than whether fixed signals predict fixed responses. Similarly, Arnold and Bar-On (2020) point out that it is important to note cases in which the same signals are responded to similarly even when produced in different contexts. One clear pattern that has emerged is that many calls appear to be less contextually specific than originally suspected (e.g., Fichtel, 2020; Fischer, 2020; Zuberbühler, 2000, 2001). This realization points to the possibility that alarm calls may be more accurately described as reflecting the emotional state of the receiver (Fichtel, 2020; Rendall et al., 2009) rather than as being referential (Seyfarth et al., 2010).

Schwartz et al. (2020) present the intriguing promise of analyzing primate screams for new insights regarding the relation between emotional arousal and acoustical information. Carlson et al. (2020) extend the work on alarm calls by exploring the idea that some species may serve as community 
informants - producing calls that affect the behavior of other species and reduce their own call rates. They examine the potential of tits to be community informants, analyzing the extent to which their calls are perceived as predator threat information by sympatric species. Although they found that most species examined did not use the information to alter their own behavior or calling, they have provided a useful system for analyzing such effects in other species, enhancing understanding of interspecific communication.

As with most dichotomies in comparative cognition, the dichotomy of information (Seyfarth et al., 2010) versus emotion signals (Rendall et al., 2009) is likely to be false (Snowdon, 2020) as is the distinction between the ensuing representation as either a categorical representation or emotion induction (Schwartz et al., 2020). Most signals likely contain some elements of both (see also Arnold \& Bar-On, 2020). Sievers and Gruber (2020) also point out that the distinction between arbitrary and non-arbitrary signals may be better conceived as a continuum. Pepperberg (2020) refers to another misleading dichotomy that was prevalent during the time period preceding these publications - that between nature and nurture reflected in the differing approaches of psychologists and biologists to the study of language evolution. This dichotomy remains despite several calls for psychologists to situate their work within the animal's natural ecology (e.g., Eaton et al., 2018; Kamil, 1987; Vonk \& Shackelford, 2012). Lyn and Christopher (2020) also stress the importance of examining the animal's rearing environment.

Whereas the most explicit inheritance of the target papers is the body of work on referential signals in alarm calls, and other types of acoustic signals, the work more broadly inspired investigations into the evolution of human language examining species as diverse as songbirds (Scully et al., 2020), squirrels (McRae, 2020) and apes (Leroux \& Townsend, 2020; Lyn \& Christopher, 2020). Seyfarth and colleagues encouraged other researchers to examine the types of categories and concepts represented by nonhumans using their natural vocal behavior. Scully and colleagues (2020) examine the extent to which chickadees categorize calls according to the season in which they were produced. Their work suggests that chickadees do not perceive seasonal calls as representing different categories. However, previous work has shown that birds do perceive songs differently based on information about the caller; for example, sex (Hahn et al., 2015) and origin (Hahn et al., 2016).

Explorations of continuities from nonhumans to humans have sometimes focused on abilities such as syntax and symbolic representation in language-trained apes (Lyn \& Christopher, 2020). However, Leroux and Townsend (2020) point out that, although there is promising data on syntacticlike structures in the communication of monkeys, comparable data from apes is lacking, which creates a disconcerting gap in the reconstruction process. Mann and Hoeschele (2020) suggest the possibility of examining even more nuanced divisions of units of communication by examining segments within breaths. They suggest that variation within segments could relate to referential and symbolic labels, such as those that evolved in human language. Sievers and Gruber (2020) attend to the arbitrariness of signals and examine the learning processes involved in the acquisition of signals. They argue that the process of learning the use and meaning of a signal can inform the extent to which signals are arbitrary and subject to social learning, which, for humans, is key to learning how to communicate effectively. Taken together, the authors of this special issue highlight various gaps in the body of literature that has emerged in the four decades since Seyfarth and colleagues presented their influential work. Schwartz et al. (2020, p. 202) identify four questions that they see emerging from a summary of recent research, each of which is centered around "factors that contribute to: (1) call usage by senders, (2) the particular within-type acoustic structure of a given call, (3) receivers' responses to different types of calls, and (4) receivers' responses to acoustic variation within call types." A focus on the pragmatics of expressive communication will also be helpful (Arnold \& Bar-On, 2020).

There are of course aspects of language evolution that have been extensively studied but were not touched on by contributors in the current volume. Gestural communication has emerged as an important form of communication in apes (Pollick \& de Waal, 2007), the study of which has replaced, to some extent, the focus on vocal production in these species (Tomasello, 2007; Tomasello \& Call, 2007). Additionally, the relationship between symbolic communication and other cognitive abilities has driven much research on the evolution of human cognition (Premack, 2004). Kolodny and Edelman (2018) 
theorized that the emergence of language was predicated on our ancestors' ability to perform sequencedependent processes, which were involved in the production of complex tools. They argued that the neural networks supporting complex, hierarchical, sequence-dependent tool production were exapted to support communication, such that these two suites of cognitive abilities were uniquely tied together in human evolution. However, others (e.g., Bolhuis et al., 2014) have suprisingly argued that language basically emerged fully formed in a "single, rapid, emergent event." In an interview with Ben James, Berwick refers to the purported connection between toolmaking and language as a useful metaphor, at best (James, 2018). But the fact remains that language is likely inextricably tied to the emergence of other adaptations that were critical to human survival and reproduction. We have yet to determine the connections on our evolutionary path, leaving room for continued exciting developments in this area.

\section{References}

Arnold, K., \& Bar-On, D. (2020). Primate pragmatics, expressive behavior, and the evolution of language. Animal Behavior and Cognition, 7, 117-130.

Boesch, C. (2020). Listening to the appeal from the wild. Animal Behavior and Cognition, 7, 257-263.

Bolhuis, J.J., Tattersall, I., Chomsky, N, Berwick, R.C. (2014), How could language have evolved? PLoS Biology, 12, e1001934.

Carlson, N. V., Healy, S. D., \& Templeton, C. N. (2020). What makes a 'community informant'? Reliability and anti-predator signal eavesdropping across mixed-species flocks of tits. Animal Behavior and Cognition, 7, 214-246.

Cheney, D. L., \& Seyfarth, R. M. (1990). Attending to behaviour versus attending to knowledge: Examining monkeys' attribution of mental states. Animal Behaviour, 40, 742-753.

Eaton, T., Hutton, R., Leete, J., Lieb, J., Robeson, A. \& Vonk, J. (2018). Bottoms-up: Rejecting top-down humancentered approaches in comparative psychology. International Journal of Comparative Psychology, 31.

Fichtel, C. (2020). Monkey alarm calling: It ain't all referential, or is it? Animal Behavior and Cognition, 7, 101107.

Fischer, J. (2020). Nonhuman primate alarm calls then and now. Animal Behavior and Cognition, 7, 108-116.

Hahn, A. H., Hoang, J., McMillan, N., Campbell, K., Congdon, J., \& Sturdy, C. B. (2015). Biological salience influences performance and acoustic mechanisms for the discrimination of male and female songs. Animal Behaviour, 104, 213-228.

Hahn, A. H., Hoeschele, M., Guillette, L. M., Hoang, J., McMillan, N., Congdon, J. V., Campbell, K. A., Mennill, D. J., Otter, K. A., Grava, T., Ratcliffe, L. M., \& Sturdy, C. B. (2016). Black-capped chickadees categorize songs based on features that vary geographically. Animal Behaviour, 112, 94-104.

James, Ben (2018, June 10). A sneaky theory of where language came from. The Atlantic, https://getpocket.com/explore/item/a-sneaky-theory-of-where-language-came-from?utm_source=pocketnewtab

Kamil, A C. (1987). A synthetic approach to the study of animal intelligence. Papers in Behavior and Biological Sciences 35, 257-308

Kolodny O., \& Edelman S. (2018). The evolution of the capacity for language: The ecological context and adaptive value of a process of cognitive hijacking. Philosophical Transactions of the Royal Society B, 373, 20170052.

Leroux, M., \& Townsend, S. W. (2020). Call combinations in great apes and the evolution of syntax. Animal Behavior and Cognition, 7, 131-139.

Lyn, H., \& Christopher, J. L. (2020). How environment can reveal semantic capacities in nonhuman animals. Animal Behavior and Cognition, 7, 159-167.

Mann, D.C., \& Hoeschele, M. (2020). Segmental units in nonhuman animal vocalization as a window into meaning, structure, and the evolution of language. Animal Behavior and Cognition, 7, 151-158.

McRae, T. R. (2020). A review of squirrel alarm-calling behavior: What we know and what we do not know about how predator attributes affect alarm calls. Animal Behavior and Cognition, 7, 168-190.

Pepperberg, I. M. (2020). Vocal communication in nonhuman animals: View from the wings. Animal Behavior and Cognition, 7, 95-100.

Pollick, A. S., \& de Waal, Frans B. M. (2007). Ape gestures and language evolution. Proceedings of the National Academy of Sciences of the United States of America, 104, 8184-8189.

Premack, D. (2004). Is language the key to human intelligence? Science, 303, 318-320. 
Rendall, D., Owren, M. J., \& Ryan, M. J. (2009). What do animal signals mean? Animal Behaviour, 78, 233-240.

Schwartz, J. W., Engelberg, J. W. M., \& Gouzoules, H. (2020). Evolving views on cognition in animal vocal communication: Contributions from scream research. Animal Behavior and Cognition, 7, 192-213.

Scully, E. N., Campbell, K. A., Congdon, J. V., \& Sturdy, C. B. (2020). Discrimination of black-capped chickadee (Poecile atricapillus) chick-a-dee calls produced across seasons. Animal Behavior and Cognition, 7, 247256.

Seyfarth, R. M., Cheney, D. L., Bergman, T., Fischer, J., Zuberbühler, K,, \& Hammerschmidt, K. (2010). The central importance of information in studies of animal communication. Animal Behaviour, 80, 3-8.

Seyfarth, R. M., Cheney, D. L., \& Marler, P. (1980a). Monkey responses to three different alarm calls: Classification and semantic communication. Science, 210, 801-803.

Seyfarth, R. M., Cheney, D. L., \& Marler, P. (1980b). Vervet monkey alarm calls: Semantic communication in a free-ranging primate. Animal Behaviour, 28, 1070-1094.

Sievers, C., \& Gruber, T. (2020). Can nonhuman primate signals be arbitrarily meaningful like human words? An affective approach. Animal Behavior and Cognition, 7, 140-150.

Snowdon, C. T. (2020). Vervet monkey alarm calls: Setting the historical context. Animal Behavior and Cognition, 7, 87-94.

Tomasello, M. (2007). If they're so good at grammar, then why don't they talk? hints from apes' and humans' use of gestures. Language Learning and Development, 3, 133-156.

Tomasello, M., \& Call, J. (2007). Ape gestures and the origins of language. In J. Call, \& M. Tomasello (Eds.), The gestural communication of apes and monkeys (pp. 221-239). Taylor \& Francis Group/Lawrence ErlbaumAssociates.

Vonk, J. \& Shackelford, T.K. (2012). Comparative evolutionary psychology: A United discipline for the study of evolved traits In. J. Vonk \& T. Shackelford (Eds). Oxford handbook of comparative evolutionary psychology (pp. 547-560). Oxford University Press.

Wheeler, B. C., \& Fischer, J. (2012). Functionally referential signals: A promising paradigm whose time has passed. Evolutionary Anthropology, 21, 195-205

Zuberbühler, K (2000). Referential labelling in Diana monkeys. Animal Behaviour, 59, 917-927.

Zuberbühler, K. (2001). Predator-specific alarm calls in Campbell's guenons. Behavioral Ecology and Sociobiology, $50,414-422$. 